\title{
Biopolitics, psychosomatics, participating bodies
}

\section{Monica Greco ${ }^{\circ}$}

\section{"Smokers and obese people 'soft targets' for NHS savings, say surgeons." 1}

The rationing of medical services to patients with so-called lifestyle diseases remains a controversial proposition worthy of newspaper headlines-like the one above from The Guardian, reporting on findings from a study commissioned in 2015 by the Royal College of Surgeons. ${ }^{2}$ Such headlines tend to prompt lavish commentary from readers, and opinions tend to be highly polarised. Even at the highest levels, public debate on these issues often appears trapped in a stark alternative between blaming individuals for their behavioural 'choices', or appealing to forms of determinism-whether biological or environmental-in order to excuse them. In the face of ageing populations and a growing prevalence of chronic conditions, there has long been a general consensus about the need to shift the focus of healthcare policy and practice towards prevention. Yet, everyday vocabularies of prevention rarely reflect what we know about the complexity of such diseases and of the behaviours associated with them. 'Both folk psychology and practical reasoning in the clinic,' write Kirmayer and Gómez-Carrillo in their contribution to this special issue, 'tend to employ simpler, often dichotomous models' that are not necessarily helpful, or indeed that may be counterproductive, potentially to the point of harm. ${ }^{3}$

Against the backdrop of this broad social and cultural context, this collection of articles aims to enrich the repertoire of discussion around questions of agency, responsibility, motivation and self-management. In recent years, these concepts have become explicitly central to the ambitions of a 'participatory' model of healthcare where patients are expected to 'shift from being mere passengers to responsible drivers of their health'. ${ }^{4}$ Interrogating them both critically and creatively appears particularly important and urgent today, at a time when the marked resurgence of an individualist political rhetoric opposes 'shirkers' and 'strivers', often questioning

Correspondence to Professor Monica Greco,

Sociology, Goldsmiths College, London SE14 6NW, UK; m.greco@gold.ac.uk benefit entitlements and the authenticity of illnesses or disabilities. Discourses that are critical of this situation, both within and outside the academy, can be forceful in advocating for patients and for the reality of their suffering, but rarely query the fundamental concepts-of causality, agency and even 'reality' itself-that frame the terms of the debate. The result can be a situation of polemical deadlock, reflecting the structure of the dichotomous model implicitly shared by both sides. $^{5}$ The intention of this special issue is to offer a measure of distance from the heat of contemporary polemics and to create room for exploring conceptual resources that might enable conversations to develop otherwise, by drawing on a richer imaginary of possibilities.

The papers in this collection stem from a set of discussions that took place in the context of a Wellcome-funded symposium held at the University of Cambridge in 2016, and of a workshop held the following year at Goldsmiths, University of London. These events brought together a group of scholars and clinicians based in multiple disciplines including philosophy, sociology, anthropology, psychology, geography, psychological medicine and psychiatry. Each coming from very different perspectives, they all received the same invitation-namely to engage with the multiple connotations of the term psychosomatic, with a view to reclaiming some of the possibilities associated with the project of a 'psychosomatic medicine'. The choice of psychosomatic (and psychosomatics) as the keynote term for the events-and now for this special issue-is not accidental. At face value, on a purely abstract level, the term encapsulates a clear ambition to challenge conceptual dichotomies, to supersede them by proposing to focus on the relation between supposedly opposite, incommensurable entities: minds and bodies, thought and matter, moral freedoms and physical necessities. But this clear and abstract connotation is not the reason for proposing the term as a starting point for discussion, quite the contrary. The choice stems rather from an empirical acknowledgement that the term psychosomatic-and the ambition of psychosomatics as a field of research-have been bedevilled by ambiguity and semantic confusion for decades. ${ }^{6}$ Calls for the word to be abandoned, in favour of supposedly more adequate ones, have been numerous and diverse-reflecting a variety of clinical, research, and vernacular settings with their specific constraints. Psychosomatic is thus a term that perplexes, or one that at least should perplex. To many, it already sounds decidedly obsolete. Put differently, psychosomatic is a term that gives pause, slowing thought down. The invitation to gather around it might be described, to cite Isabelle Stengers' paraphrase of Donna Haraway, as an invitation to 'stay with troubling words', for how these can help to challenge established habits of thought. $^{\text {' }}$

Staying with the word psychosomatic for a moment, then, let us consider a set of contrasts that characterise its uses in contemporary Western culture and medicine. On the one hand, we have an interdisciplinary field of scientific research called 'psychosomatic medicine'-with its conferences, professional associations, journals, all of which carry the word psychosomatic in the title. It is a wide-ranging field that encompasses clinical, epidemiological, as well as experimental research, combining concepts and evidence from disciplines as diverse as epigenetics, endocrinology, immunology and neurology, anthropology, psychoanalysis and psychology, cybernetics. It is worth stressing explicitly that the scope of psychosomatic medicine is not limited to any particular category of illnesses or diseases: the official journal of the American Psychosomatic Societycalled Psychosomatic Medicine-publishes research on the genesis of cancer alongside research on chronic fatigue syndrome (CFS), diabetes, the healing of wounds, and everything in between. It is also worth stressing that psychosomatic medicine is not a new field: it has developed internationally since the early decades of the 20th century, and has gone through many changes between then and now, reflecting broader transformations in science, culture and society. Over this time, and taken as a whole, this field has produced a wealth of conceptual tools and propositions that allow us to articulate how culturally mediated social relations might get 'under our skin' to produce both physical and mental ill-health.

What I have just described, however, is not what most people think of when they hear the word psychosomatic, or when they use it in everyday life. In the practice of medicine, at least in anglophone societies (the situation differs in continental Europe, most notably in Germany), 
psychosomatic is hardly ever used. ${ }^{8}$ When it is used colloquially, its connotations tend to be offensive. A study published in the BMJ in 2002 set out to measure its offensiveness compared with other diagnostic expressions, by interviewing new patients attending a general neurology clinic in Edinburgh. ${ }^{9}$ Psychosomatic scored as the third most offensive expression of the 10 surveyed, after 'all in the mind' and 'hysterical'. To call an illness psychosomatic carries connotations of moral failure and is taken to imply that the illness is imaginary, fake or inauthentic, possibly even intentional. In everyday use the adjective also implies that there are other, more authentic illnesses, to which it does not apply. ${ }^{10}$ The fact that psychosomatic can be a stigmatising label is of immediate significance to individuals whose illness might be characterised in this way, and who understandably reject it for this reason. Today, more than ever before, those who are wary of stigmatising the sick, of blaming the victim, steer well clear of the term psychosomatic.

Last but not least, through its ostensible emphasis on the role of psyche at the expense of broader sociocultural dimensions of health and pathology, the term psychosomatic has been associated with a set of specifically modern, Western assumptions about the nature of minds, bodies and of medical problems. These include, but are not limited to, the tendency to regard pathology as localised within individuals; and a fundamental assumption that biological processes remain invariant across cultures and indeed constitute the source of human universals. Both assumptions have been challenged by the work of scientists and anthropologists working cross-culturally, some of whom have proposed the term 'sociosomatic' as a supplement to psychosomatic in order to mark this limitation. ${ }^{11}$ A different but related dissatisfaction with psychosomatic is apparent in the preference for the term 'biopsychosocial' as the descriptor of a 'new medical model'. ${ }^{12}$

Why then stay with psychosomatic, this troublesome word? Why not reach for less ambiguous, less compromised, more comprehensive alternatives? The value of doing so becomes readily apparent as soon as psychosomatic is approached not as the name for a (new) medical modelthat is, as the answer to a range of clinical and scientific puzzles-but rather as an indicator of the underlying problematic itself. In other words, while psychosomatic may be inadequate as the name for a solution, it points straight to the heart of the problem that has animated a host of partial, never definitive, ever more creative quests for solutions. In this sense, it is an excellent term. The problematic of psychosomatics is indeed modern and Western: this is not an effect of bias, but simply of the fact that the problematic has an objective, historical, situated existence. This problematic is intimately tied to a specific version of dualism that became established through the knowledge practices of modern science, and that went on to provide the structure for a full-fledged mode of existence, leaving no aspect of modern life -biological, social, cultural, economic, political-untouched. The problem of psychosomatics can then be understood as a symptom, or as the way in which concrete aspects of experience that fall through the cracks of modern dualist solutions make themselves felt: in routine clinical encounters with illnesses that remain 'unexplained', for example, all the way to the laboratory, haunted by the spectre of never-quite-controllable placebo effects. ${ }^{13}$

As a symptom of the shortcomings of dualism, however, the problem of psychosomatics is more than a purely negative state of affairs, waiting to be fixed. Its generative power is evident in the proliferation of all the forms of knowledge, practice and experience that have emerged in response to it. In an ongoing and unfinished process, these transform and diversify the problem of psychosomatics in an abstract sense, as well as the world concretely. This rather more positive appreciation of the nature of 'problems' as creative and generative - is not limited to psychosomatics and indeed applies more generally. ${ }^{14}$ In the problematic of psychosomatics, however, the re-evaluation of the value of problems becomes reflexive and explicit. For example, one of the politically more daring and interesting contributions of the tradition of psychosomatic medicine is the proposition that illness may be regarded as an adaptive form of self-regulation (where the problem constitutes, in effect, a solution), or even an expression of creative vitality akin to a work of art. ${ }^{15}$ More than just representational, such propositions are themselves self-consciously creative, acting as lures for different futures at both a clinical-therapeutic and at an epistemic level.

The papers that comprise this special issue of BMJ Medical Humanities all speak, in their rich diversity, to various aspects of the problematic of psychosomatics. The initial steer was provided by the first paper in the collection, a draft of which was circulated in advance of the second encounter of the group. This paper revisits classic approaches to 'psychosomatic problems', which typically set out by denouncing the conceptual inadequacy of mind/body dualism, to propose the resilience of dualism as an alternative starting point for analysis and discussion. The argument traces this resilience to the far-reaching sociomaterial efficacy of the epistemic configuration set in place by the development of modern science, which A $\mathrm{N}$ Whitehead referred to as the 'bifurcation of nature'. It then links Whitehead's proposition to Foucault's account of (neo) liberal political economy through the concepts of biopolitics and biopolitical forms of governance. This provides the basis for two further operations: one is to reclaim the relevance of specific propositions from the history of psychosomatic medicine that, like Whitehead, placed the question of value at the centre of their problematisation of dualism. The second is the outline of an invitation to consider recent discourses of participatory medicine in terms of the (typically bifurcated) assumptions at work in them, and to imagine what the concept of 'participation' might involve from a non-bifurcated perspective. Such a perspective might include bodies alongside minds as meaningful agents expressing preferences and values, and the paper refers to it as participation all the way down.

The invitation to explore the concept of participation all the way down is taken up most directly and adventurously by Martin Savransky in his contribution, where he draws on pragmatist and speculative philosophies-alongside the science of slime moulds and ant colonies-to flesh out this proposition in infinitesimal detail, to the scale of unicellular organisms and beyond. The paper sets out by asking: 'how to characterise what a "participant" is? Who and what is capable of participation?' It then goes on to develop a form of pluralistic panpsychism involving a double proposition: on the one hand, that 'all things think', that is, all things involve and express 'varying degrees and forms of sensory awareness, purposes and aims, the discernment of relevance, problem-solving, decision-making and feeling'; and, on the other hand, that any one participant is always already multiple and internally divergent. Savransky thus articulates the possibility that embodied human beings are capable of unruly and subversive forms of participation precisely to the extent that, even as individuals, they always already embody multiple and divergent perspectives. 
The contribution by Duschinsky, Messina and Reisz similarly speaks to the theme of how vital processes always exceed biopolitical capture. The focus in this paper is on the negotiations that subjects entertain with biopolitics under situations of 'structurally induced attrition'. The paper pivots around the contrast between Lauren Berlant's account of Mary Gaitskill's novel, Two Girls, Fat and Thin, and an ethnographic account of processes of burn-out in investment banking associates. In the former, different ways of engaging (or not) in embodied activities that are fundamental sites of self-governance-such as eating and sex-serve to produce affects that make life momentarily more bearable, by offering respite from the 'burden of subjectivity, through the participation of bodily processes'. The second account illustrates how bodily cues are ignored in pursuit of normative modes of subjectivation in a work-intensive, results-driven industry, only to irrupt back on the scene - as burn-out - in ways that are 'so predictable that they form part of the expectable arc' of career trajectories. Based on these two examples, the paper offers poignant reflections on the concept of coping, and on the trade-offs individuals make between making life bearable in the short term and thriving in the longer term.

The theme of bodies-in-environments is further developed in the contribution by Brown and Reavey, which focuses on how different spaces facilitate or hinder 'feelings of being alive' that are transversal to existing diagnostic categories. The paper conjugates a theoretical analysis of the concept of 'vitality', based on the work of Frederic Worms among others, with a discussion of how space functions in both inpatient and community-based mental healthcare as a 'relational nexus of bodies, materials, affects and signs', through which different qualities and degrees of 'aliveness' can be experienced. In conclusion, the authors offer a set of suggestions on how the concept of 'vitality' can be operationalised for a research agenda on vital spaces.

Michael Schillmeier returns to the 'bifurcation of nature' as a central theme in his contribution, to argue for the fundamental relevance of value to the understanding of 'all bodies and things-human and non-human alike'. The paper begins by offering a critique, not of the biopsychosocial model per se, but of recent calls to make the model more impactful by foregrounding research on the neurology of mentalising processes, and therefore by adapting its language to that of biomedicine. This and other theoretical strategies, the paper argues, repeat the gesture of bifurcation that the biopsychosocial model had set out to overcome. To counteract this tendency, Schillmeier proposes to radicalise the biopsychosocial model by 'generalising the importance of psychosocial value processes and societal modes of organising them' . Drawing on the sociological monadology of Gabriel Tarde to develop a 'cosmopolitics of existence', the paper offers an account of bodies as 'value experiences' and argues for the relevance of subjectivity to every mode of existence, including that of inorganic bodies.

The turn to neuroscience in psychosomatic research comes centre-stage in the paper by Felicity Callard and Stan Papoulias. Here the authors critically examine the promise of an emergent field of research-that of neuropsychoanalysis-to 'instal subjective intentionality within rather than alongside biology', and thereby to offer an alternative to standard, bifurcated models of mind and body. Focusing on how neuropsychoanalysis translates the Freudian concept of the drive as a series of Basic Emotion Systems, the paper demonstrates how the field imagines the mental apparatus to be 'of the same order as ... the functional systems of the body', rendering drives as subordinate to demands of biological survival, and resituating non-adaptive aspects of the Freudian psyche within the confined domain of addiction as a special case. Neuropsychoanalysis thus betrays the radical potential of psychosomatics-and indeed of psychoanalysis-by 'sequestering fantasy from biology', and ultimately by instituting a too-easy distinction between what constitutes health and what constitutes pathology.

Addiction is the topic of the contribution by Darin Weinberg, which sets off by offering a detailed review of the landscape of addiction science and public policy. His elegant account demonstrates the continued entrenchment of dichotomous models of explanation based on an alternative between biological reductionism and liberal voluntarism. These, Weinberg argues, share a tendency 'to think of subjectivities, minds and/or bodies as singular and unified'. In contrast, the paper goes on to argue for the importance of addressing the multiplicity of the body as 'host to psychosomatically diverse desiring subjectivities', and to consider addiction ecologically, that is, as a function of the sociomaterial, reciprocal relations between agents and environments, 'without presuming the humanity of the agent in question'. This approach can offer a better understanding of the unstable dispositions of addicts, which are strongly associated with the tendency to blame them for their fates.

In the paper that concludes the main section of the special issue, Laurence Kirmayer and Ana Gómez-Carrillo examine the forms and consequences of dichotomous reasoning about illness causation in everyday situations and clinical practice. Differentiating between ontological, epistemic and moral versions of dualism, they argue that all three are at play in biomedicine and 'serve to rationalise, stabilise, and reinforce each other', in ways that make it difficult for models that challenge the terms of any one form of dualism to become established. Focusing on the problem of agency, the paper then moves on to argue for the value of '4-E' cognitive science as a bridge between phenomenology and mechanistic explanations, through which a discredited biopsychosocial model might be renewed. This approach is richly illustrated through the example of Resignation Syndrome among refugee children in Sweden. In their conclusion, the authors reflect on some reasons why ecosocial, integrative approaches remain difficult to realise in practice. Interestingly, these reasons include the notion that our own cognitive apparatus has a preference for binary categories, and the fact that 'the social world pushes back with moral dichotomies'.

Faithful to the intention that has animated this collective project from the very start, namely that of enriching the semantic repertoire of discussions around questions of agency and responsibility in relation to health, this special issue could not fail to include a paper addressed to the public perception of the problematic of psychosomatics. In their paper on Chronic Fatigue Syndrome (CFS) and an illness-focused approach to care, Michael Sharpe and Monica Greco discuss the controversy surrounding the provision of cognitive and behavioural treatments to patients with CFS. The paper argues that the concepts of 'illness' and 'disease'-typically used to mark the difference between the subjective experience of symptoms and objective evidence of pathology-have very different moral connotations. Patients who have illnesses without evidence of disease find themselves in an epistemically paradoxical and morally ambiguous predicament, which may explain the strong antagonism towards illness-focused treatments expressed by some. To overcome the persistence and the consequences of these moral connotations, 
the paper argues, the epistemic paradox must be acknowledged, and strategies for managing the paradox in daily life should be explored. In the longer term, transforming the moral connotations of illnesses-without-disease requires the cultivation of non-bifurcated ways of articulating the difference between disease and illness.

The articles collected in this special issue focus on different aspects of the problematic of psychosomatics and do so in a variety of ways. Prompted by the problematic itself, however, all of them seek to develop perspectives that avoid the bifurcation of nature and that reject the epistemic divisions of labour along disciplinary lines that the bifurcation implies. The special issue taken as a whole thus contributes to the project of a medical humanities explicitly engaged in questions of ontology. ${ }^{16}$ As such, it is a clear invitation for scholars in the field to regard themselves as active contributors to the understanding of vital processes that defy and subvert the distinction between 'nature' and 'culture'.

Funding The authors have not declared a specific grant for this research from any funding agency in the public, commercial or not-for-profit sectors.

Competing interests None declared.

Patient consent for publication Not required.

Provenance and peer review Not commissioned; internally peer reviewed.

(C) Author(s) (or their employer(s)) 2019. No commercial re-use. See rights and permissions. Published by BMJ.

\section{Check for updates}

To cite Greco M. Med Humanit 2019;45:103-106.

Accepted 23 May 2019

Published Online First 13 June 2019

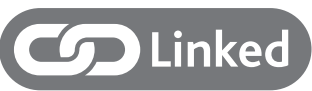

- http://dx.doi.org/10.1136/medhum-2018-011588

Med Humanit 2019;45:103-106. doi:10.1136/ medhum-2019-011717

\section{NOTES}

1. Denis Campbell (2016), "Smokers and obese people 'soft targets' for NHS savings, say surgeons," The
Guardian, https://www.theguardian.com/society/2016/ apr/22/smokers-obese-people-soft-targets-nhs-savingssurgeons

2. Royal College of Surgeons (2016), Smokers and Overweight Patients: Soft Targets for NHS Savings? (London: RCS)

3. See contribution by Sharpe and Greco in this special issue. See also Nortin Hadler (1996), "If You Have to Prove You Are III, You Can't Get Well: The Object Lesson of Fibromyalgia," Spine 21, no 20: 2397-2400.

4. Society for Participatory Medicine, "About Us," Society for Participatory Medicine, accessed September 2, 2018, http://participatorymedicine.org/about

5. Monica Greco (2012), "The Classification and Nomenclature of 'Medically Unexplained Symptoms': Conflict, Performativity, and Critique," Social Science and Medicine 75, no 12: 2362-2369.

6. Zbigniew J. Lipowski (1984), "What Does the Word 'Psychosomatic' Really Mean? A Historical and Semantic Inquiry," Psychosomatic Medicine 46, no 2: 153-171.

7. Isabelle Stengers (2017), "Staying With Troubling Words," Groupe d'Etudes Constructivistes, April 2017, accessed May 5, 2019, https://groupeconstructiviste. files.wordpress.com/2017/04/stengers_staying-withtroubling-words.pdf

8. Stephan Zipfel et al. (2016), "Psychosomatic Medicine in Germany: More Timely Than Ever," Psychotheraphy and Psychosomatics 85: 262-269.

9. Jon Stone et al. (2002), "What Should We Say to Patients with Symptoms Unexplained by Disease? The 'Number Needed to Offend'," BMJ 325 (December 21-28, 2002): 1449-1450.

10. B Lask (1996), "Psychosomatic Medicine' Not 'Psychosomatic Disorders," Journal of Psychosomatic Research 40, no 5: 457-460.

11. A Kleinman and A E Becker (1998), "'Sociosomatics': The Contribution of Anthropology to Psychosomatic Medicine," Psychosomatic Medicine 60, no 4: 389-393; see also the volume edited by James Wilce, 2003, Social and Cultural Lives of Immune Systems (London and New York: Routledge).

12. G L Engel (1977), "The Need for a New Medical Model: A Challenge for Biomedicine," Science 196, no 4286: 129-136.

13. Elizabeth A. Wilson (2008), "Ingesting Placebo," Australian Feminist Studies 23, no 55: 31-42.

14. On problems and problematics as generative, see "Experiments On the Problematic", a forthcoming special issue of Theory, Culture, and Society edited by Martin Savransky (forthcoming in 2019).

15. See Monica Greco (2017), "Thinking with Outrageous Propositions," in Speculative Research: The Lure of Possible Futures, ed. Alex Wilkie, Marsha Rosengarten and Martin Savransky (London and New York: Routledge).

16. William Viney et al. (2015), Felicity Callard and Angela Woods, "Critical Medical Humanities: Embracing
Entanglement, Taking Risks," Medical Humanities, 41, no 1: 2-7.

\section{BIBLIOGRAPHY}

Campbell, Denis, The Guardian. "Smokers and obese people 'soft targets' for NHS savings, say surgeons.", 2016. https://www.theguardian.com/society/2016/apr/ 22/smokers-obese-people-soft-targets-nhs-savingssurgeons

Engel, G.L. "The need for a new medical model: a challenge for biomedicine." Science 196, no. 4286 (1977): 129-36.

Greco, Monica. "The classification and nomenclature of 'medically unexplained symptoms': conflict, performativity and critique." Social Science \& Medicine 75, no. 12 (2012): 2362-9.

Greco, Monica. "Thinking with Outrageous Propositions." In Speculative Research: The Lure of Possible Futures, edited by A, Wilkie, M, Rosengarten, and M, Savransky. London and New York: Routledge, 2017.

Hadler, Nortin. "If you have to prove you are ill, you can't get well. The object lesson of fibromyalgia." Spine 21, no. 20 (1996): 2397-400.

Kleinman, AA E Becker. " "Sociosomatics": the contributions of anthropology to psychosomatic medicine." Psychosomatic medicine 60, no. 4 (1998): 389-93.

Lask, Bryan. "Psychosomatic medicine" not "psychosomatic disorders." Journal of Psychosomatic Research 40, no. 5 (1996): 457-60.

Lipowski, Zbigniew J. "What does the word "psychosomatic" really mean? A historical and semantic inquiry." Psychosomatic Medicine 46, no. 2 (1984): 153-71.

Royal College of Surgeons. Smokers and Overweight Patients: Soft Targets for NHS Savings? London: RCS, 2016.

Society for Participatory Medicine. "About Us". Accessed 2 Sep 2018. http://participatorymedicine.org/about.

Stengers, Isabelle. "Staying With Troubling Words. Groupe d'Etudes Constructivistes.", 2017. Accessed 5 May 2019. https://groupeconstructiviste.files.wordpress. com/2017/04/stengers_staying-with-troubling-words. pdf

Stone, Jon, Wojtek Wojcik, Daniel Durrance, Alan Carson, Steff Lewis, Lesley MacKenzie, Charles P Warlow, and Michael Sharpe. "What should we say to patients with symptoms unexplained by disease? The "number needed to offend"." BMJ 325, no. 7378 (2002): 1449-50.

Viney, William, Felicity Callard, and Angela Woods. "Critical medical humanities: embracing entanglement, taking risks." Medical Humanities 41, no. 1 (2015): 2-7.

Wilce, James. Social and Cultural Lives of Immune Systems. London and New York: Routledge, 2003.

Wilson, Elizabeth A.. "Ingesting placebo." Australian Feminist Studies 23, no. 55 (2008): 31-42.

Zipfel, Stephan, Wolfgang Herzog, Johannes Kruse, and Peter Henningsen. "Psychosomatic medicine in Germany: more timely than ever." Psychotherapy and Psychosomatics 85, no. 5 (2016): 262-9. 